\title{
Crescimento intra-uterino restrito diagnosticado pelo índice ponderal de Rohrer e sua associação com morbidade e mortalidade neonatal precoce
}

\author{
Intrauterine growth retardation diagnosed by Rohrer's ponderal index and its \\ association with morbidity and early neonatal mortality
}

Adehilde Maria Martins Santos ${ }^{1}$, Ana Claire Pimenteira Thomaz ${ }^{2}$, José Elias Soares da Rocha ${ }^{3}$

\begin{abstract}
RESUMO
Objetivo: diagnosticar o crescimento intra-uterino restrito (CIUR) por meio do índice ponderal de Rohrer e sua associação com morbidade e mortalidade neonatal precoce. Métodos: estudo retrospectivo, descritivo e de coorte transversal, no qual foram incluídos 2741 recém-nascidos (RN), sendo 2053 casos de grávidas hígidas, 228 de pré-eclâmpsia leve (PE), 52 com PE grave, 25 com PE que evoluiu para eclâmpsia, 136 de ruptura prematura das membranas (RPM) e 247 de tabagistas. O índice ponderal (IP) de Rohrer foi calculado segundo a equação: $\mathrm{IP}=$ peso/estatura $^{3}$ x 100 e se utilizaram os valores 2,25 e 3,10 dos percentis 10 e 90 de Lubchenco. Classificou-se como CIUR assimétrico aquele $\mathrm{RN}$ com IP $<2,25$ e peso inferior ao percentil 10, como simétrico, IP entre 2,25 e 3,10 e peso inferior ao percentil 10, ao passo que adequado para idade gestacional (AIG) aquele com IP entre 2,25 e 3,10 e peso entre os percentis $10 \mathrm{e} 90$. O estudo estatístico foi realizado pelo teste $t$ não pareado, teste não paramétrico do $\chi^{2}$ e teste exato de Fisher, considerado significante valor de $\mathrm{p}<0,05$. Resultados: o baixo peso $(<2500 \mathrm{~g})$ ao nascer incidiu em 3,6\% $(100 / 2741)$ dos casos, ao passo que a taxa de CIUR diagnosticado pelo IP foi de 15,7\% (430/2741), sendo 14,0\% assimétricos e 1,7\% simétricos. A taquipnéia transitória foi a complicação mais freqüente $(8,3 \%)$ entre os assimétricos, seguido da asfixia $(5,7 \%)$ e infecção $(2,6 \%)$. A taquipnéia transitória incidiu em $6,5 \%$ dos simétricos, seguido da asfixia $(4,3 \%)$, síndrome de aspiração de mecônio $(2,2 \%)$, hipoglicemia (2,2\%) e infecção (2,2\%). O óbito neonatal precoce foi semelhante entre os RN com CIUR e AIG, ambos alcançando cifra de $0,3 \%$. Conclusões: o índice ponderal de Rohrer diagnosticou os diferentes padrões de crescimento intra-uterino, os quais não seriam reconhecidos utilizando-se o peso em função da idade gestacional. Os $\mathrm{RN}$ assimétricos apresentaram maior taxa de taquipnéia transitória e de asfixia, entretanto sem significância estatística em relação às demais modalidades de crescimento intrauterino. A taxa de óbito neonatal precoce foi semelhante entre os RN assimétricos e os AIG.
\end{abstract}

PALAVRAS-CHAVE: Retardo do crescimento fetal; Mortalidade fetal; Complicações na gravidez; Peso-estatura; Antropometria; Morbidade

\section{ABSTRACT}

Purpose: to diagnose intrauterine growth restriction (IGR) and its connection with early neonatal morbidity and mortality, through Roher's ponderal index (PI). Methods: this was a retrospective, descriptive study of transversal cohort, in which 2741 newborns (NB) were included, 2053 of them from healthy pregnant women, 228 from women with mild pregnancyrelated hypertension, 52 from those with severe pregnancy-related hypertension, 25 from those with mild pregnancy-related hypertension that evolved to eclampsia, 136 from those with premature membrane rupture, and 247 from women who smoked along gestation. Roher's PI was calculated by the equation: PI = weight/height ${ }^{3} \mathrm{x} 100$ and the values 2.25 and 3.10 of Lubchenco's 10 and 90 percentiles were used to classify the types of IGR. IGR was classified as asymmetric for NB with $\mathrm{PI}<2.25$ and weight lower than percentile 10, as symmetric, with PI from 2.25 to 3.10 and weight lower than percentile 10, and adequate for gestational age with PI from 2.25 to 3.10, and weight from 10 to 90 percentiles. Statistical analysis was performed using the non-paired $t$ test, the non-parametric $\chi^{2}$ test and Fisher's exact test, with significance set at a value of $\mathrm{p}<0.05$. Results: low birth weight $(<2,500 \mathrm{~g})$ was present in 3.6\% $(100 / 2741)$ of the cases, while the rate of IGR diagnosed through PI was $15.7 \%$ (430/2741), 14.0\% being asymmetric and $1.7 \%$ symmetric. The most frequent complication among the asymmetric IGRNB was transient tachypnea (8.3\%), followed by asphyxia (5.7\%) and infection (2.6\%). Transient tachypnea was present in $6.5 \%$ of symmetric IGRNB, followed by asphyxia (4.3\%), meconium aspiration syndrome (2.2\%), hypoglycemia $(2.2 \%)$ and infection $(2.2 \%)$. Early neonatal death was similar for NB with restricted IGR and adequate IGR for gestational age, both groups reaching a rate of $0.3 \%$. Conclusions: Rohrer's PI was able to diagnose the different IGR patterns, which would not be known if the birth weight had been calculated in terms of gestational age. The asymmetric NB presented a

1 Médica Pediatra da Casa Maternal Santa Mônica da Universidade de Ciências da Saúde de Alagoas (UNCISAL) Maceió (AL) - Brasil.

2,3 Professor Adjunto Doutor do Departamento de Tocoginecologia e Pediatria da Universidade Federal de Alagoas - UFAL - Maceió (AL) - Brasil. Correspondência: José Elias Soares da Rocha

Rua Dr. Artur Machado nº 07 - Fernão Velho - 57070040 - Maceió - AL - Telefone: (82) 3788333 - e-mail: jelsrocha@yahoo.com.br

Recebido em: 13/12/2004 Aceito com modificações em: 11/3/2005

Rev Bras Ginecol Obstet. 2005;27(6):303-9 
higher incidence of transient tachypnea and asphyxia, without statistical significance in relation the other IGR patterns. The frequency of early neonatal death was similar for the asymmetric and adequate for gestational age NB groups.

KEYWORDS: Fetal growth retardation; Fetal mortality; Pregnancy complications; Height weight; Anthropometry; Morbidity

Introdução

A nutrição intra-uterina adequada é condição impar para o crescimento e desenvolvimento normais do feto, constituindo fator primordial para a determinação do peso ao nascer ${ }^{1-4}$. Nos países em desenvolvimento, o nascimento de crianças com peso menor que 2.500 gramas é considerado problema de saúde pública ${ }^{5}$, aspecto que faz do peso ao nascer o determinante mais importante da morbidade e mortalidade infantil, inclusive exercendo influência até a vida adulta, sendo considerado forte indicador do desenvolvimento econômico e social ${ }^{6,7}$.

Baixo peso ao nascer é definido, pela Organização Mundial de Saúde $(\mathrm{WHO})^{8}$, como todo recém-nascido (RN) com peso inferior a 2.500 gramas, independentemente da idade gestacional. Há dois tipos de RN de baixo peso: o pré-termo $(<37$ semanas) e aquele de termo ( $\geq 37$ semanas), os quais não seriam distinguidos entre si considerando apenas o peso, mas após a construção de curvas de crescimento fetal, levando em conta o peso ao nascer e a idade gestacional ${ }^{9,10}$, esses RN puderam ser diferenciados. Com a utilização dessas curvas de crescimento fetal, foi possivel definir crescimento intra-uterino restrito (CIUR) como aquele RN que se encontra abaixo do $10^{\circ}$ percentil para a idade gestacional e como adequado para a idade gestacional (AIG) aquele que fica entre o $10^{\circ}$ e o $90^{\circ}$ percentil, ao passo que aqueles acima do $90^{\circ}$ percentil são grandes para a idade gestacional (GIG) ${ }^{9,10}$. Por sua vez, os RN com CIUR podem ser classificados em três categorias: simétrico, assimétrico e intermediário. O CIUR simétrico, também denominado hipoplásico, intrínseco ou proporcional, é decorrente da ação de fatores etiológicos que atuam no início da gravidez, isto é, na fase de "hiperplasia celular", reduzindo o número de células de todos os órgãos. Esses RN têm pior prognóstico em relação aos assimétricos, são mais suscetiveis às ações mórbidas no decorrer da gestação e durante o parto ${ }^{11-13}$. Os principais fatores etiológicos desse tipo de CIUR são as infecções pelo vírus da rubéola, citomegalovirus, HIV, herpes, etc., toxoplasmose materna aguda, sífilis recente, tuberculose e cromossomopatias ${ }^{14-17}$. Por sua vez, o assimétrico ou desproporcional tem como agente etiológico fatores que atingem a gravidez no início do $3^{\circ}$ trimestre, com déficit no crescimento celular (hipotrofia celular) ${ }^{18,19}$, fato que origina a desproporção observada entre o tamanho da cabeça (que permanece com o volume das células normais) e do corpo (que sofre redução no volume celular), representado principalmente pela redução da circunferência abdominal, visto que o figado é um dos órgãos mais afetados, caracterizando os RN magros com estatura normal e pólo cefálico proporcionalmente grande ${ }^{18,19}$. O CIUR intermediário ou misto sofre ação dos agentes causadores nas duas fases de crescimento celular, a de hiperplasia e a de hipertrofia. Os principais agentes são representados pela desnutrição da grávida, bem como o consumo de substâncias como drogas ilícitas, álcool, tabaco, cafeína, etc. ${ }^{13}$.

O padrão de crescimento intra-uterino pode ser diagnosticado utilizando-se apenas o peso ao nascer ${ }^{20}$ ou, mais freqüentemente, associando peso e estatura que, utilizado pela primeira vez por Rohrer, recebeu a denominação de índice ponderal (IP), representado pela fórmula: IP = peso/estatu$\mathrm{ra}^{3} \times 100^{21}$. A utilização do IP permite diagnosticar as diferentes modalidades de restrição do crescimento intra-uterino, que não seria possivel se a variável utilizada fosse apenas o peso ao nascer ${ }^{10,22}$.

O IP fetal também foi possivel ser determinado a partir da década de oitenta, principalmente com o desenvolvimento tecnológico da ecografia de tempo real, mensurando-se o diâmetro biparietal, circunferência craniana, circunferência abdominal, comprimento do fêmur e relações entre si, com boa acurácia no diagnóstico do CIUR quando comparado ao IP neonatal ${ }^{23}$.

Por ser o IP fetal passivel de interpretações errôneas, conseqüentes à variação biológica fetal associada a uma sensibilidade e especificidade sofrivel $^{23}$, e pelo fato de a utilização do IP neonatal ter custo zero e ser de fácil reprodutibilidade, além de permitir o diagnóstico e a condução dos RN a curto, médio e longo prazo diante dos diferentes padrões de crescimento intra-uterino observa$\operatorname{dos}^{22}$, optamos por utilizar o IP neonatal neste trabalho. Portanto, os objetivos do presente estudo foram diagnosticar o CIUR por meio do IP de Rohrer e verificar sua associação com morbidade e mortalidade neonatal precoce.

\section{Métodos}

O presente estudo foi retrospectivo, descritivo, de coorte transversal, no qual foram incluí- 
dos 2741 RN atendidos no setor de Neonatologia da Maternidade Escola Santa Mônica da Universidade de Ciências da Saúde de Alagoas (MESM UNCISAL), assim distribuídos: 2053 de grávidas hígidas, 228 de portadoras de pré-eclâmpsia (PE) leve, 52 com $\mathrm{PE}$ grave e 25 de $\mathrm{PE}$ que evoluíram para eclâmpsia, 136 de gestantes com ruptura prematura das membranas (RPM) e 247 de gestantes tabagistas. O estudo foi aprovado previamente pelo Comitê de Ética em Pesquisa da Universidade Federal de Alagoas (UFAL).

A seleção de pacientes obedeceu aos seguintes critérios de inclusão: idade gestacional de termo (37 a 41 semanas e 6 dias); as mães dos RN que constituíram o grupo de hígidas não deveriam ser portadoras de doenças durante a gestação e/ ou outras condições como gravidez gemelar e RN malformados; os RN do grupo de PE não apresentavam nenhuma outra doença ou condição mórbida associada à sindrome hipertensiva, ao passo que os RN de gestantes com RPM não estavam submetidos a nenhuma outra doença associada; por sua vez, os RN de gestantes tabagistas foram submetidos somente aos efeitos do tabaco, sendo excluídos aqueles cujas mães relataram o consumo de outras drogas.

O diagnóstico de RN de gestante hígida foi considerado quando a mesma cursava toda gravidez sem apresentar sinais/sintomas de doenças que poderiam exercer influência sobre as trocas gasosas e nutritivas entre mãe e concepto no espaço intervilositário.

A PE foi diagnosticada obedecendo ao seguinte critério: 1) PE forma leve: grávidas com pressão diastólica $<100 \mathrm{mmHg}$ durante o último trimestre da gestação, associada a edema e proteinúria $\geq 300$ $\mathrm{mg} / 24$ horas. 2) PE forma grave: niveis tensionais diastólicos $\geq 110 \mathrm{mmHg}$, associado à presença de edema e proteinúria $\geq 2$ g em 24 horas, associado a sinais e sintomas como mal-estar precordial, dor no hipocôndrio direito, cefaléia, escotomas, icterícia, etc. 3) Eclâmpsia: grávidas com hipertensão, edema e proteinúria que apresentaram convulsões do tipo tônico-clônico e/ou coma. 4) RN de gestantes tabagistas foram aqueles cujas mães fumaram $\geq 10$ cigarros / dia durante toda gestação, afastandose outros tipos de drogas lícitas ou ilícitas. 5) O diagnóstico de RPM foi considerado positivo quando havia relato no prontuário de perda líquida franca por via vaginal, observada ao exame com especulo e/ ou algum relato de teste ferning positivo com ou sem complemento da ecografia.

Além dos critérios de inclusão acima citados, as mães dos RN dos diferentes grupos apresentavam características semelhantes como: cor, estado civil, idade, peso, estatura e idade gestacional na resolução da gravidez.
A idade gestacional foi calculada em semanas utilizando-se a data do último periodo menstrual e, nos casos em que não constavam anotadas, considerou-se a idade gestacional obtida por meio da ecografia realizada no $1^{\circ}$ trimestre e, não havendo relato de exame ecográfico, utilizou-se o indice de Capurro somático para esse cálculo.

Os RN foram avaliados por meio de anotações dos seus respectivos prontuários, segundo rotina do setor de Neonatologia, incluindo-se idade gestacional, peso ao nascer, estatura, índice de Apgar no $1^{\circ}$ e $5^{\circ}$ minuto, morbidade e mortalidade neonatal precoce. O diagnóstico de morbidade neonatal precoce, inicialmente, foi baseado na presença de sinais patológicos previamente estabelecidos: asfixia, hipoglicemia, infecção, taquipnéia transitória e sindrome de aspiração de mecônio. Quando necessário, exames complementares como: raios-X, glicemia, hemograma, culturas e hemoculturas foram levados em consideração na complementação diagnóstica. A mortalidade neonatal precoce teve como critério diagnóstico a identificação do óbito do RN até o sétimo dia de vida, observado no prontuário do nascituro.

As medidas antropométricas mensuradas foram as seguintes: peso ao nascer e estatura. $\mathrm{O}$ peso ao nascer foi obtido utilizando-se balança pesa-bebê Filizola, com sensibilidade para $10 \mathrm{~g}$, aferida semanalmente. No momento da pesagem, o RN era colocado na balança sem roupas. A estatura foi obtida utilizando-se fita métrica adaptada por Díaz et al. ${ }^{24}$, no Centro Latino Americano de Perinatologia e Desenvolvimento Humano (CLAP), segundo orientação da OMS e Organização Panamericana de Assistência à Saúde (OPAS), usando-se como pontos de referência a superficie da cabeça e a planta do pé, com o RN em posição supina.

O cálculo do IP foi realizado a partir da equação originalmente proposta por Rohrer: $\mathrm{IP}=\mathrm{P} / \mathrm{E}^{3} \mathrm{x}$ 100 , onde $\mathrm{P}=$ peso do $\mathrm{RN}$ em gramas $(\mathrm{g})$ e $\mathrm{E}=$ estatura em cm e, para classificar o crescimento intrauterino, utilizaram-se os valores de 2,25 e 3,10 dos percentis 10 e 90 da curva de crescimento de Lubchenco et al. ${ }^{25}$. Classificou-se como CIUR assimétrico aquele $\mathrm{RN}$ com $\mathrm{IP}<2,25$ e peso inferior ao percentil 10 , como simétrico, IP entre 2,25 e 3,10 e peso inferior ao percentil 10, ao passo que AIG aquele com IP entre 2,25 e 3,10 e peso entre os percentis 10 e 90.

A análise estatística foi realizada utilizandose o teste $t$ não pareado para comparar médias e o teste não paramétrico do $\chi^{2}$ com correção de Yates e o teste exato de Fischer para comparar as variáveis dependentes. A significância estatística foi considerada com valor de $\mathrm{p}<0,05$. 


\section{Resultados}

Foram incluídas 2741 gestantes e seus RN que constituíram a casuística do presente estudo.

A Tabela 1 mostra a distribuição das pacientes e seus RN em seis grupos: hígidas, PE forma leve, $\mathrm{PE}$ forma grave, $\mathrm{PE}$ que evoluiu para eclâmpsia, tabagistas e RPM, e suas associações com parâmetros demográficos maternos, variáveis inerentes à gestação, ao parto e resultados neonatais. O peso médio dos RN de mulheres tabagistas e com RPM foram inferiores aos demais grupos, mostrando diferença estatística significante $(\mathrm{p}<0,05)$.

Tabela 1 - Distribuição dos grupos de mães hígidas, tabagistas e com doenças da gravidez e seus recém-nascidos, segundo dados demográficos e parâmetros relacionados com a gravidez, o parto e resultados neonatais.

\begin{tabular}{|c|c|c|c|c|c|c|c|}
\hline \multirow[b]{2}{*}{ Parâmetros } & \multicolumn{5}{|c|}{ Grupos } & \multirow[b]{2}{*}{ RPM } & \multirow[b]{2}{*}{ Total } \\
\hline & Hígidas & PE (leve) & PE (grave) & PE (eclâmpsia) & Tabagistas & & \\
\hline $\mathrm{n}$ & 2053 & 228 & 52 & 25 & 247 & 136 & 2741 \\
\hline Idade materna (anos) (média $\pm D P$ ) & $22,0 \pm 5,5$ & $23,0 \pm 6,1$ & $23,0 \pm 7,7$ & $20,0 \pm 6,2$ & $25,0 \pm 6,1$ & $23,0 \pm 5,7$ & \\
\hline Estatura materna $(m)$ (média $\pm D P)$ & $1,54 \pm 0,05$ & $1,57 \pm 0,05$ & $1,56 \pm 0,11$ & $1,56 \pm 0,11$ & $1,60 \pm 0,05$ & $1,57 \pm 0,06$ & \\
\hline Peso materno $(\mathrm{Kg})$ (média $\pm \mathrm{DP}$ ) & $56,6 \pm 9,8$ & $59,5 \pm 9,1$ & $64,6 \pm 6,1$ & $65,0 \pm 6,0$ & $58,5 \pm 9,0$ & $58,8 \pm 8,2$ & \\
\hline Solteiras (\%) & 64,2 & 56,6 & 51,9 & 68 & 70 & 54,4 & \\
\hline Casadas (\%) & 35,8 & 43,4 & 48,1 & 32 & 30 & 45,6 & \\
\hline IG na resol. grav. (sem., média $\pm D P$ ) & $39,4 \pm 1,4$ & $39,3 \pm 1,4$ & $39,0 \pm 1,4$ & $38,6 \pm 1,6$ & $39,4 \pm 1,3$ & $39,1 \pm 1,4$ & \\
\hline 5 cons. pré-natal $(\%)$ & 55,5 & 66,6 & 57,7 & 60 & 38,9 & 63,2 & \\
\hline Parto transvaginal $(\%)$ & 70,6 & 24,6 & 3,8 & 16 & 76,5 & 39 & \\
\hline Parto cesáreo (\%) & 29,4 & 75,4 & 96,2 & 84 & 23,5 & 61 & \\
\hline Peso ao nascer $(\mathrm{g})$ (média $\pm \mathrm{DP}$ ) & $3.278 \pm 456$ & $3.334 \pm 523$ & $3.323 \pm 520$ & $3.243 \pm 600$ & $3.174 \pm 431^{*}$ & $3.190 \pm 410^{* *}$ & \\
\hline \multicolumn{8}{|l|}{ Índice de Apgar (média土DP) } \\
\hline $1^{0}$ minuto & $7,9 \pm 1,5$ & $7,7 \pm 1,5$ & $7,9 \pm 1,2$ & $7,7 \pm 1,9$ & $8,0 \pm 1,4$ & $7,8 \pm 1,5$ & \\
\hline $5^{0}$ minuto & $9,1 \pm 0,8$ & $9,0 \pm 0,8$ & $9,1 \pm 0,7$ & $8,8 \pm 1,3$ & $9,2 \pm 0,7$ & $9,0 \pm 1,3$ & \\
\hline Índice ponderal (média $\pm D P$ ) & $2,50 \pm 0,3$ & $2,60 \pm 0,3$ & $2,60 \pm 0,3$ & $2,60 \pm 0,3$ & $2,50 \pm 0,4$ & $2,50 \pm 0,3$ & \\
\hline
\end{tabular}

n: número de pacientes por grupo; PE: pré-eclâmpsia; RPM: ruptura prematura das membranas; DP: desvio padrão; IG: idade gestacional; m: metros; g: gramas; kg: quilograma; resol: resolução; grav.: gravidez; cons.: consultas; sem.: semanas; * $p=0,02 ;{ }^{* *} p=0,0007$

O baixo peso ao nascer $(<2.500 \mathrm{~g})$ foi diagnosticado em 100 casos $(3,6 \%)$ do presente estudo, sendo mais freqüente entre os RN de mulheres tabagistas, 5,7\% (14/247), e de eclâmpsia, $16,0 \%$ (4/25). Entretanto, foi observada significância apenas com o grupo de eclâmpsia em relação ao grupo hígido $(\mathrm{p}<0,05)$.

A Tabela 2 mostra a distribuição da morbimortalidade neonatal precoce entre os RN de mulheres dos grupos estudados. A maioria dos RN, cerca de $80 \%$, ficaram isentos de condições mórbidas, excetuando-se os grupos com eclâmpsia e de RPM, que apresentaram cifras de 60 e 63,2\%, respectivamente. $\mathrm{O}$ grupo de $\mathrm{RN}$ de mulheres que tiveram eclâmpsia apresentou o seguinte contingente mórbido: três $(12 \%)$ casos de asfixia (sem significância; p>0,05), seis (24\%) de taquipnéia transitória (com significância; $p<0,05$ ) e um $(4 \%)$ óbito neonatal precoce (sem significância; $p>0,05$ ).
A taquipnéia transitória incidiu em sete $(13,5 \%)$ casos no grupo de $\mathrm{PE}$ grave sem significância ( $p>0,05)$. O grupo oriundo da RPM apresentou 16 $(11,8 \%)$ casos de taquipnéia transitória (sem significância; p>0,05) e $26(19,1 \%)$ de infecção, com significância estatística $(\mathrm{p}<0,05)$. Por sua vez o grupo hígido e o tabagista apresentaram quatro $(0,2 \%)$ e dois $(0,8 \%)$ casos de óbitos neonatais precoces, respectivamente, sem diferença significante $(p>0,05)$.

Os padrões de crescimento intra-uterino diagnosticados pelo IP de Rohrer estão distribuídos na Tabela 3. Considerando o total de RN do estudo, verificou-se que o CIUR incidiu em $15,7 \%$ dos casos $(430 / 2741)$, sendo $384(14,0 \%)$ do tipo assimétrico e $46(1,7 \%)$ do tipo simétrico. No grupo de RN hígidos, não se observou diferença significativa ( $p>0,05)$, quanto à freqüência, entre os AIG e aqueles com restrição de crescimento simétrico e 
assimétrico. O mesmo ocorreu entre os grupos de $\mathrm{PE}$ leve e os de tabagistas. A associação dos RN segundo o IP e o peso ao nascer, encontra-se na Tabela 4. A comparação entre a classe de peso em relação ao índice ponderal mostrou que os RN de baixo peso $(<2.500 \mathrm{~g})$ apresentaram significativamente $(\mathrm{p}<0,05)$ maiores taxas, 54\% (54/100), de CIUR assimétrico do que os pequenos para a idade gestacional (PIG) (2.500 - $3.000 \mathrm{~g})$, com taxa de 25,9\% (184/711), AIG (3.001-3.999 g), com 7,7\% (146/1906), e macrossômicos, com 0,0\% (0/24). Considerando apenas os RN de baixo peso e o tipo de CIUR, observou-se que o assimétrico foi mais prevalente, $14 \%$ (54/384), do que o simétrico, $2 \%$ (46/2295), com diferença significativa $(p>0,05)$ quando comparados. A Tabela 5 associa o tipo de crescimento intra-uterino com a morbidade neonatal precoce. Podemos verificar que em relação à ausência de sinais mórbidos não houve diferença significativa $(p>0,05)$ entre o grupo de RN AIG quando comparado aos grupos com CIUR simétrico e assimétrico. A taquipnéia transitória foi o sinal mórbido mais freqüente, presente em 200 (7,3\%) casos entre os RN com os diferentes tipos de CIUR e os não portadores de CIUR, sendo que aqueles classificados como portadores de restrição de crescimento assimétrico apresentaram maior freqüência $(32=8,3 \%)$, seguido do AIG $(166=7,2 \%)$, do simétrico $(3=6,5 \%)$ e pelo GIG $(2=3,2 \%)$, sem diferença significante $(p>0,05)$. A asfixia incidiu em 156 $(5,7 \%)$ casos do total de RN, não havendo diferença entre o grupo de RN assimétrico $(22=5,7 \%)$ e o AIG $(130=5,8 \%)$. Índices ainda menores foram observados nos grupos com CIUR simétrico $(2=4,3 \%)$ e no GIG $(2=3,2 \%)$. Por sua vez a infecção incidiu em 50 $(1,8 \%)$ casos, não havendo diferenças significativas $(p>0,05)$ entre as freqüências dos grupos de CIUR assimétrico $(10=2,6 \%)$, simétrico $(1=2,2 \%)$ e AIG $(39=1,7 \%)$.

Considerando o óbito no período neonatal precoce entre os diferentes padrões de crescimento intra-uterino desse estudo, não foi observada diferença significante $(p>0,05)$ entre os $0,3 \%(6 /$ 2269) do grupo AIG contra os $0,3 \%(1 / 384)$ do grupo de RN com CIUR assimétrico.

Tabela 2 - Distribuição dos recém-nascidos de mulheres hígidas, tabagistas e com doenças da gravidez, segundo a morbidade e mortalidade neonatal precoce.

\begin{tabular}{|c|c|c|c|c|c|c|c|c|c|c|c|c|c|c|c|c|}
\hline \multirow[t]{4}{*}{ Grupo } & \multicolumn{10}{|c|}{ Morbimortalidade } & & & & & & \\
\hline & \multicolumn{6}{|c|}{ Ausente } & \multicolumn{4}{|c|}{ Presente } & & & & & & \\
\hline & \multirow[t]{2}{*}{$\mathrm{n}$} & \multirow[t]{2}{*}{$\%$} & \multicolumn{2}{|c|}{ ASF } & \multicolumn{2}{|c|}{ SAM } & \multicolumn{2}{|c|}{$\pi$} & \multicolumn{2}{|c|}{ HG' } & \multicolumn{2}{|c|}{ INF } & \multicolumn{2}{|c|}{ Óbito } & \multicolumn{2}{|c|}{ Total } \\
\hline & & & $\mathrm{n}$ & $\%$ & $\mathrm{n}$ & $\%$ & $\mathrm{n}$ & $\%$ & $\mathrm{n}$ & $\%$ & $\mathrm{n}$ & $\%$ & $\mathrm{n}$ & $\%$ & $\mathrm{n}$ & $\%$ \\
\hline Hígida & 1775 & 85,5 & 115 & 5,6 & 10 & 0,5 & 146 & 7,1 & 2 & 0,1 & 21 & 1,0 & 4 & 0,2 & 2053 & 100 \\
\hline PE leve & 189 & 82,9 & 17 & 7,5 & 1 & 0,4 & 18 & 7,9 & 1 & 0,4 & 2 & 0,9 & 0 & 0,0 & 228 & 100 \\
\hline PE grave & 42 & 80,8 & 2 & 3,8 & 1 & 1,9 & 7 & 13,5 & 0 & 0,0 & 0 & 0,0 & 0 & 0,0 & 52 & 100 \\
\hline Eclâmpsia & 15 & 60,0 & 3 & 12,0 & 0 & 0,0 & $06^{*}$ & 24,0 & 0 & 0,0 & 0 & 0,0 & 1 & 4,0 & 25 & 100 \\
\hline Tabagista & 217 & 87,8 & 13 & 5,3 & 2 & 0,8 & 11 & 4,5 & 0 & 0,0 & 2 & 0,8 & 2 & 0,8 & 247 & 100 \\
\hline RPM & 86 & 63,2 & 8 & 5,9 & 0 & 0,0 & 16 & 11,8 & 0 & 0,0 & $26^{* *}$ & 19,1 & 0 & 0,0 & 136 & 100 \\
\hline Total & 2324 & 84,8 & 158 & 5,8 & 14 & 0,5 & 204 & 7,4 & 3 & 0,1 & 51 & 1,9 & 7 & 0,2 & 2741 & 100 \\
\hline
\end{tabular}

ASF: asfixia; SAM: síndrome de aspiração de mecônio ; TT: taquipnéia transitória; HG: hipoglicemia; INF: infecção; PE: pré-eclâmpsia; RPM: ruptura prematura das membranas; * $p=0,01$; ${ }^{* *} p<0,001$.

Tabela 3 - Distribuição dos recém-nascidos de mulheres hígidas, tabagistas e com doenças da gravidez, segundo os padrões de crescimento intra-uterino.

\begin{tabular}{ccccc}
\hline Grupo & \multicolumn{4}{c}{ Crescimento intra-uterino } \\
& $\begin{array}{c}\text { Restrito } \\
\text { Restrito }\end{array}$ & AIG $\quad$ GIG & Total \\
assimétrico & simétrico & & &
\end{tabular}

\begin{tabular}{lrrrrrrrrrr} 
& \multicolumn{1}{c}{$\mathbf{n}$} & \multicolumn{1}{c}{$\%$} & \multicolumn{1}{c}{$\mathbf{n}$} & \multicolumn{1}{c}{$\%$} & $\mathbf{n}$ & $\%$ & $\mathbf{n}$ & $\%$ & $\mathbf{n}$ & $\%$ \\
\hline Hígida & 300 & 78,1 & 26 & 56,5 & 1685 & 74,9 & 42 & 67,7 & 2053 & 74,9 \\
PE leve & 23 & 6,0 & 5 & 11,0 & 190 & 8,4 & 10 & 16,1 & 228 & 8,3 \\
PE grave & 4 & 1,0 & 2 & 4,3 & 42 & 1,9 & 4 & 6,5 & 52 & 1,9 \\
Eclâmpsia & 3 & 0,8 & 2 & 4,3 & 17 & 0,8 & 3 & 4,8 & 25 & 0,9 \\
Tabagista & 31 & 8,1 & 8 & 17,4 & 206 & 9,2 & 2 & 3,2 & 247 & 9,0 \\
RPM & 23 & 6,0 & 3 & 6,5 & 109 & 4,8 & 1 & 1,6 & 136 & 5,0 \\
Total & 384 & 100 & 46 & 100 & 2249 & 100 & 62 & 100 & 2741 & 100 \\
\hline
\end{tabular}

PE: pré-eclampsia; RPM: Ruptura prematura das membranas; AIG: Adequado para a idade gestacional; GIG: Grande para a idade gestacional.
Tabela 4 - Distribuição dos recém-nascidos de mulheres hígidas, tabagistas e com doenças da gravidez, segundo o peso ao nascer e 0 índice ponderal.

\begin{tabular}{|c|c|c|c|c|c|c|c|c|}
\hline \multicolumn{9}{|c|}{ Índice ponderal } \\
\hline \multirow{2}{*}{$\begin{array}{l}\text { Peso } \\
\text { (gramas) }\end{array}$} & \multicolumn{2}{|c|}{$<2,25$} & \multicolumn{2}{|c|}{$2,25-3,10$} & \multicolumn{2}{|c|}{$>3,10$} & \multicolumn{2}{|c|}{ Total } \\
\hline & $\mathrm{n}$ & $\%$ & $\mathrm{n}$ & $\%$ & $\mathrm{n}$ & $\%$ & $\mathrm{n}$ & $\%$ \\
\hline$<2.500$ & $54^{*}$ & 54,0 & 46 & 46,0 & 0 & 0,0 & 100 & 100 \\
\hline $2.500-3.000$ & 184 & 25,9 & 523 & 73,5 & 4 & 0,6 & 711 & 100 \\
\hline $3.001-3.999$ & 146 & 7,7 & 1708 & 89,6 & 52 & 2,7 & 1906 & 100 \\
\hline$\geq 4.000$ & 0 & 0,0 & 18 & 75,0 & 6 & 25,0 & 24 & 100 \\
\hline Total & 384 & 14,0 & 2295 & 83,7 & 62 & 2,3 & 2741 & 100 \\
\hline
\end{tabular}


Tabela 5 - Distribuição dos recém-nascidos de mulheres hígidas, tabagistas e com doenças da gravidez, segundo o crescimento intra-uterino e a associação com a morbidade neonatal precoce.

\begin{tabular}{|c|c|c|c|c|c|c|c|c|c|c|}
\hline \multirow[t]{3}{*}{ Morbidade } & \multicolumn{8}{|c|}{ Crescimento intra-uterino } & & \\
\hline & \multicolumn{2}{|c|}{$\begin{array}{c}\text { Restrito } \\
\text { assim. }\end{array}$} & \multicolumn{2}{|c|}{$\begin{array}{c}\text { Restrito } \\
\text { sim. }\end{array}$} & \multicolumn{2}{|c|}{ AIG } & \multicolumn{2}{|c|}{ GIG } & \multicolumn{2}{|c|}{ Total } \\
\hline & $\mathrm{n}$ & $\%$ & $\mathrm{n}$ & $\%$ & $\mathrm{n}$ & $\%$ & $\mathrm{n}$ & $\%$ & $\mathrm{n}$ & $\%$ \\
\hline Ausente & 318 & 82,8 & 38 & 82,6 & 1903 & 84,6 & 58 & 93,6 & 2317 & 84,5 \\
\hline Asfixia & 22 & 5,7 & 2 & 4,3 & 130 & 5,8 & 2 & 3,2 & 156 & 5,7 \\
\hline SAM & 2 & 0,5 & 1 & 2,2 & 11 & 0,5 & 0 & 0 & 14 & 0,5 \\
\hline TTRN & 32 & 8,3 & 3 & 6,5 & 163 & 7,2 & 2 & 3,2 & 200 & 7,3 \\
\hline Hipoglicemia & 0 & 0,0 & 1 & 2,2 & 3 & 0,1 & 0 & 0 & 4 & 0,1 \\
\hline Infecção & 10 & 2,6 & 1 & 2,2 & 39 & 1,7 & 0 & 0 & 50 & 1,8 \\
\hline Total & 384 & 100 & 46 & 100 & 2249 & 100 & 62 & 100 & 2741 & 100 \\
\hline
\end{tabular}

AIG: Adequado para a idade gestacional; GIG: Grande para idade gestacional; Assim. Assimétrico: Sim. Simétrico

SAM: Síndrome de aspiração de mecônio; TTRN: Taquipnéia transitória.

\section{Discussão}

O presente estudo mostrou freqüência de CIUR diagnosticado pelo IP de Rohrer de 15,7\%, sendo $14,0 \%$ assimétricos e 1,7\% simétricos, evidenciando taxa 4,4 vezes maior do que os $3,6 \%$ de RN com baixo peso ao nascer, fato que revela a possibilidade de desnutrição intra-uterina entre RN com peso AIG. Esse achado se torna relevante principalmente por se tratar de gestação de termo, cuja média de idade gestacional foi $39 \pm 1,5$ semanas. O percentual de $7,7 \%$ de CIUR do tipo assimétrico observado entre os RN AIG é semelhante aos $8,0 \%$ da casuística de Walter e Ramaekers ${ }^{26}$, destacando-se que em os ambos estudos os RN desnutridos apresentaram pesos adequados (entre 3.001 g e 3.999 g). Por outro lado, é inferior àquela de Caiza Sánchez et al. ${ }^{22}$, que foi de $13,0 \%$. A restrição de crescimento entre RN com essas características ponderais tem sido descrita na literatura como aquele concepto que não atingiu o potencial máximo de crescimento e clinicamente é reconhecido como RN que apresenta deficiência em tecidos moles ${ }^{1}$.

Portanto, diagnosticaram-se neste estudo: 25,9\% de RN PIG, 2,7\% AIG com IP aumentado (baixa estatura genética), $75 \%$ de GIG com IP normal (grande constitucional) e 7,7\% de RN AIG com IP baixo (CIUR subclínico), que jamais seriam diagnosticados se tivéssemos utilizado apenas o peso ao nascer como meio diagnóstico do crescimento intra-uterino, fato que destaca a utilidade do IP para essa finalidade, pois ao associar o peso à estatura, aumenta-se a sensibilidade do método ${ }^{22}$. Saliente-se ainda que, mesmo sendo um índice antropométrico, existe independência em relação ao sexo e raça ${ }^{26,27}$.

Considerando a incidência de $1,7 \%$ de CIUR simétrico no presente estudo contra $14,0 \%$ do assimétrico, vê-se que o tipo de restrição intrauterina não está de acordo com os achados de pesquisas realizadas em países pobres da América Latina, cuja maior incidência foi de RN simétri$\cos ^{22,28}$, fato que revela o perfil de país desenvolvido no achado desse estudo.

A taxa de baixo peso ao nascer entre os RN de mulheres que tiveram eclâmpsia foi cinco vezes maior em relação àqueles de mulheres hígidas, aspecto que inclusive lhes conferiu restrição do crescimento e elevada taxa de taquipnéia transitória em relação aos demais grupos. A possivel associação do descolamento prematuro da placenta normalmente inserida aos casos de PE, bem como a presença de espasmo vascular inerente a essa doença, reduzindo a perfusão placentária, é fator primordial na gênese da morbidade e mortalidade perinatal $^{19}$, podendo explicar o resultado ominoso encontrado entre esses RN. O percentual de 17,4\% de CIUR simétrico observado entre os RN de mulheres tabagistas pode ser explicado pelo fato de o tabaco consumido durante a gestação ser fator preponderante na redução da troca de nutrientes e oxigênio entre mãe e feto, conseqüência, dentre outras, da diminuição do contingente de vilosidades coriônicas, reduzindo assim a população de capilares vilositários necessários para as trocas entre o compartimento materno e o fetal $^{29}$.

A taxa de $19,1 \%$ de infecção neonatal imediata encontrada entre os RN de RPM foi extremamente elevada em relação aos demais grupos, entretanto, é obviamente esperada, pois se trata de rupturas com periodo de latência prolongado, fato que predispõe à infecção ascendente ${ }^{30}$.

O baixo peso ao nascer teve maior influência como determinante do tipo de CIUR, visto que o assimétrico foi preponderante em relação ao simétrico neste grupo. O perfil predominante de CIUR assimétrico observado nesse estudo não pode ser atribuído exclusivamente às doenças que incidem no último trimestre da gravidez, uma vez que $78,1 \%$ dos RN com CIUR assimétrico eram filhos de mulheres hígidas. Este aspecto destaca o IP de Rohrer como método antropométrico simples e de fácil aplicação no diagnóstico dos diferentes padrões de CIUR, possibilitando, assim, a condução adequada dos RN no período neonatal precoce, diante das possiveis condições mórbidas que se associam a esses desvios do crescimento intra-uterino, proporcionando, portanto, maior probabilidade de sobrevivência e melhor qualidade de vida a esses RN. 


\section{Referências}

1. Gruenwald P. Growth of the human fetus. I. Normal growth and its variation. Am J Obstet Gynecol. 1966;94(8):1112-9.

2. Battaglia FC, Lubchenco LO. A practical classification of newborn infants by weight and gestational age. J Pediatr. 1967;71(2):159-63.

3. Kramer MS, Olivier M, McLean FH, Dougherty GE, Willis DM, Usher RH. Determinants of fetal growth and body proportionality. Pediatrics. 1990;86(1):18-26.

4. Lang U, Baker RS, Khoury J, Clark KE. Effects of chronic reduction in uterine blood flow on fetal and placental growth in the sheep. Am J Physiol Regul Integr Comp Physiol. 2000;279(1):R53-9.

5. Villar J, Belizan JM. The timing factor in the pathophysiology of the intrauterine growth retardation syndrome. Obstet Gynecol Surv. 1982;37(8):499-506.

6. Anderson GD, Blidner IN, McClemont S, Sinclair JC. Determinants of size at birth in a Canadian population. Am J Obstet Gynecol. 1984;150(3):23644.

7. Wilcox AJ. On the importance and unimportance of birthweight. Int J Epidemiol. 2001;30(6):1233-41.

8. World Health Organization. Public health aspects of low birth weight: third report of the Expert Committee on Maternal and Child Health. Geneva: WHO; 1961. (WHO Technical Reporter Service, 217).

9. Lubchenco LO, Hansman C, Dressler M, Boyd E. Intrauterine growth as estimated from live born birth-weight data at 24 to 42 weeks of gestation. Pediatrics. 1963;32:793-800.

10. Matheus M, Sala MA. Curvas do indice ponderal no decorrer da gestação. J Bras Ginecol. 1990;100(3/4):59-61.

11. Daikoku NH, Johnson JW, Graf C, Kearney K, Tyson $\mathrm{JE}$, King TM. Patterns of intrauterine growth retardation. Obstet Gynecol. 1979;54(2):211-9.

12. Woods DL, Malan AF, Heese HV. Patterns of retarded fetal growth. Early Hum Dev. 1979;3(3):257-62.

13. Bitar RE. Crescimento intra-uterino retardado. In: Zugaib M, editor. Medicina fetal. 2a ed. São Paulo: Atheneu; 1998. p. 358-76.

14. Kramer MS, McLean FH, Olivier M, Willis DM, Usher RH. Body proportionality and head and length "sparing" in growth-retarded neonates: a critical reappraisal. Pediatrics. 1989;84(4):717-23.

15. Knox GE. Influence of infection on fetal growth and development. J Reprod Med. 1978;21(6):352-8.

16. Lin CC, Evans MI, editors. Intrauterine growth retardation: pathophysiology and clinical management. New York: McGraw-Hill; 1984.
17. William O. O recém-nascido com RCIU. In: Queenan JT, editor. Gravidez de alto risco. 2a ed. São Paulo: Manole; 1987. p. 511-7.

18. Hadlock FP. Avaliação ultra-sonográfica do crescimento fetal. In: Callen $P$, editor. Ultrasonografia em obstetrícia e ginecologia. 3a ed. Rio de Janeiro: Guanabara Koogan; 1996. p. 124-38.

19. Cunningham FG, Gant NF, Leveno KJ, Gilstrap III LC, Hauth JC, Wenstrom KD. Williams obstetrics. 21st ed. New York: McGraw-Hill; 2001. p. 743-64.

20.Haggarty P, Campbell DM, Bendomir A, Gray ES, Abramovich DR. Ponderal index is a poor predictor of in utero growth retardation. BJOG. 2004;111(2):113-9.

21.Dombrowski MP, Berry SM, Johnson MP, Saleh AA, Sokol RJ. Birth weight-length ratios, ponderal indexes, placental weights, and birth weightplacenta ratios in large population. Arch Pediatr Adolesc Med. 1994;148(5):508-12.

22. Caiza Sánchez ME, Diaz Rosselló JL, Simini F. Índice ponderal para calificar a una población de recién nacidos a término. An Pediatr (Barc). 2003;59(1):48-53.

23.Vintzileos AM, Lodeiro JG, Feinstein SJ, Campbell WA, Weinbaum PJ, Nochimson DJ. Value of fetal ponderal index in predicting growth retardation. Obstet Gynecol. 1986;67(4):584-8.

24. Díaz JL, Estol P, Martell M. Guias para la organización y normatización de la asistencia neonatal. Montevideo: Centro Latino Americano de Perinatologia y Desarrollo Humano; 1985. (Publicación científica, 1028).

25.Lubchenco LO, Hansman C, Boyd E. Intrauterine growth in length and head circumference as estimated from live births at gestational ages from 26 to 42 weeks. Pediatrics. 1966;37(3):403-8.

26. Walter FJ, Ramaekers LH. The ponderal index as a measure of the nutritional status at birth and its relation to some aspects of neonatal morbidity. J Perinat Med. 1982;10(1):42-7.

27. Miller HC, Hassanein K. Diagnosis of impaired fetal growth in newborn infants. Pediatrics. 1971;48(4):511-22.

28.Villar J, Altobelli L, Kestler E, Belizan J. A health priority for developing countries: the prevention of chronic fetal malnutrition. Bull World Health Organ. 1986;64(6):847-51.

29. Rocha JES, Matheus M, Sala MA. Effect of cigarette smoke on human placenta morphometry. Int $\mathrm{J}$ Gynaecol Obstet. 1998;62(3):237-42.

30. Rocha JES, Tomaz ACP, Rocha DB, Bezerra AF, Lopes ALC, Breda AMO, et al. Morbidade materna e morbimortalidade perinatal associada à infecção ascendente na rotura prematura das membranas. Rev Bras Ginecol Obstet. 2002;24(1):15-20. 